\title{
Usage of MODIS and VIIRS Combined Data for Forest Fires Area Estimation Experience
}

\author{
Ivan V. Balashov, Mikhail A. Burtsev, Andry A. Proshin, Alexey A. Matveev, Alexey A. Mazurov, \\ Konstantin S. Senko \\ Space Research Institute, Russian Academy of Sciences, Moscow, Russia \\ ivbalashov@d902.iki.rssi.ru
}

\begin{abstract}
Before Suomi NPP and NOAA-20 spacecrafts launch in 2011 and 2017, the information from the MODIS device installed on Aqua and Terra satellites was primarily used in the information systems of satellite fire monitoring for operational quantitative characteristics of natural fires (including areas covered by fire). After launching the Suomi-NPP and NOAA-20 satellites with the VIIRS device on board, it became possible to use additional data for the wildfires characteristics estimation. . This article is devoted to review the usage experience of combined MODIS and VIIRS data for estimating of the forest fires areas. The main results of the combined fires dataset usage are given and conclusions are formulated.
\end{abstract}

Keywords: VIIRS, MODIS, fires monitoring, satellite monitoring, remote sensing, NPP, NOAA-20. 


\title{
ОПЫТ СОВМЕСТНОГО ИСПОЛЬЗОВАНИЯ ДАННЫХ MODIS И VIIRS ДЛЯ ОЦЕНКИ ПЛОЩАДЕЙ ПРОЙДЕННЫХ ЛЕСНЫМИ ПОЖАРАМИ
}

\author{
И.В. Балашов, М.А. Буриев, А.А. Прошин, А.М. Матвеев, А.А. Мазуров, К.С. Сенько \\ Институт космических исследований РАН, Москва, Россия \\ ivbalashov@d902.iki.rssi.ru
}

До запуска космических аппаратов Suomi NPP и NOAA-20 в 2011 и 2017 годах в информационных системах спутникового мониторинга пожаров для оперативной количественной характеристик природных пожаров (в том числе, площадей, пройденных огнем) преимущественно использовались данные прибора MODIS, установленного на аппаратах Aqua и Terra. После запуска аппаратов Suomi-NPP и NOAA-20 с прибором VIIRS на борту стало возможным использовать при оценках характеристик природных пожаров дополнительные данные. Настоящая работа посвящена анализу опыта совместного использования данных MODIS и VIIRS для оценки площадей, пройденных лесными пожарами. Приводятся результаты вычисления основных показателей совместного использования данных разных приборов при формировании пожаров и формулируются выводы.

Ключевые слова: VIIRS, MODIS, мониторинг пожаров, спутниковый мониторинг, NPP, NOAA-20

\section{Введение}

В течение длительного времени в различных системах спутникового мониторинга пожаров, в том числе, ИСДМ-Рослесхоз [1,2], Вега [3,4], Вега-Приморье [5], для оперативной оценки пройденных огнем площадей использовалась только информация на основе обработки данных прибора MODIS, установленного на космических аппаратах Aqua и Terra. Информация, поступающая от этих систем позволяет достаточно хорошо оценивать различные характеристики природных пожаров, в первую очередь, проводить оценку площадей, пройденных огнем [6]. Благодаря открытому доступу к оперативным и архивным продуктам обработки спутниковых данных с информацией о горячих точках, а также программному обеспечению для их получения по исходным данным, продукты MODIS были практически безальтернативным источником оперативных данных о природных пожарах. В 2011 и 2017 годах были запущены аппараты Suomi NPP и NOAA 20 с прибором VIIRS [7] на борту, который обеспечил получение более детальной информации (375 м/пиксель по сравнению с 1000 м/пиксель приборов MODIS) в спектральных каналах, необходимых для выявления температурных аномалий. Кроме этого, стали доступны сервисы предоставления продуктов с информацией о пожарах и программные пакеты обработки, также являющиеся открытыми и бесплатными. Благодаря этому стало возможным широкое использование этих данных в системах дистанционного мониторинга пожаров совместно с информацией, все еще получаемой с приборов MODIS.

Дополнительное использование данных VIIRS влияет на две значимых характеристики наблюдаемых с их помощью пожаров: время первого детектирования и минимальную площадь детектирования. В настоящей работе проводится оценка качественных и количественных изменений в системах учета природных пожаров, связанных с включением информации о пожарах, получаемых на основе данных VIIRS. 


\section{Источники данных о пожарах}

Основным источником оперативных данных о температурных аномалиях достаточно высокого пространственного разрешения является прибор MODIS [8], установленный на аппараты Aqua и Terra. Прибор обеспечивает съемку земной поверхности в 36 спектральных каналах с разрешением от 250 до 1000 метров на пиксель. Для детектирования активного горения используются каналы с пространственным разрешением 1000 метров. Практически каждая точка на территории России наблюдается аппаратами Aqua и Terra не менее четырех раз в сутки. Для детектирования температурных аномалий («горячих точек») по данным прибора MODIS в настоящее время используется порогово-контекстуальный алгоритм MOD14 [9]. Пространственный размер детектированной «горячей точки» по данным MODIS составляет 1 км, однако, фактическая площадь детектированного горения может быть в несколько раз меньше и в системах, разработанных в ИКИ РАН, определяется специальным коэффициентом коррекции, сформированным на основе статистических данных о повреждениях растительности [10]. В системах мониторинга природных пожаров, разработанных и поддерживаемых в ИКИ PAH, для обработки данных алгоритмом MOD14 используется программный комплексе NASA IPOPP [11]. Такие системы обработки реализованы в центрах ФГБУ «НИЦ «Планета» [12]. Также системы мониторинга природных пожаров позволяют получать и усваивать данные о детектированных «горячих» точках предоставляемые центром FIRMS NASA [13].

Новые данные, влияние которых на характеристики детектируемых пожаров исследуется в настоящей статье, формируются на основе обработки данных прибора VIIRS (Visible Infrared Imaging Radiometer Suite) [7]. Прибор имеет 22 спектральных канала с пространственным разрешением от 375 метров на пиксель (I-bands) до 750 метров (M-bands). VIIRS установлен на космических аппаратах Suomi NPP и GPSS NOAA-20. Данные аппараты имеют близкие с Аqua и Terra орбитальные параметры. Каждая точка на территории Росиии также наблюдается с аппаратами Suomi NPP и NOAA 20 Aqua и Terra не реже четырех раз в сутки. Алгоритм выделения температурных аномалий по данным VIIRS также основан на алгоритме MOD14.

В информационных системах спутникового мониторинга пожаров, создаваемых в ИКИ РАН, одной из основных задач является оценка показателей, связанных с площадью активного горения пожара, площадью, пройденной пожаром и его длительностью. При этом, отдельный пожар может быть значительно протяженным в пространстве и гореть достаточно долго. Чтобы получать показатели площадей для таких пожаров, нельзя просто суммировать площади пикселей отдельных наблюдений. Для оценки площади на каждый интересующий момент времени информацию об отдельных «горячих точках» требуется агрегировать и пространстве, и во времени. Для этого в системах мониторинга пожаров реализована специальная технология ведения базы данных контуров пожаров [14]. Технология основана на том, что по мере поступления новых данных наблюдений по району действия пожара они добавляются к ранее сформированному состоянию пожара. При этом, имеются три степени агрегации полигона пожара:

1) отдельные горячие точки по сеансу;

2) пространственное объединение горячих точек в объект «наблюдение по сеансу»;

3) объединение наблюдения по сеансу с объединенной предыстории наблюдений в зоне пожара в состояние пожара.

Таким образом, «горячие точки», полученные по отдельным наблюдениям пожара, формируют непрерывный объект - пожар, имеющий интервал действия и область горения. В базе данных хранится вся информация о каждом состоянии развития пожара включая информацию об алгоритме и приборе, по данным которых было зарегистрировано отдельное наблюдение. Это позволяет, в частности, провести расчет и анализ статистик не только по площадям и временам детектирования, но и приборам, зарегистрировавшим пожар.

Целью настоящей работы является анализ того, насколько повлияет на оценку различных характеристик пожаров (в том числе площадей, пройденных огнем) включению в систему учета в дополнение к информации, полученной на основе приборв MODIS, информации, получаемой на основе данных приборов VIIRS. 
В ходе сравнительного анализа было необходимо ответить на вопросы:

- насколько вырастет в абсолютном значении количество пожаров при добавлении источника данных по VIIRS;

- сколько процентов пожаров будет детектироваться раньше и насколько а абсолютных значениях при включении в данных VIIRS в процедуры расчета пожаров;

- как изменятся общие площадные характеристики пройденных огнем площадей;

- насколько достоверными являются данные VIIRS в целом.

Для проведения анализа была использована база данных о пожарах, образованная данными обработки MODIS и VIIRS за пожароопасный сезон 2018 года на территорию Российской Федерации (данные получены, в частности, из Центра коллективного пользования «ИКИМониторинг» [15], обеспечивающего работу сервисов созвездия ВЕГА [4]). Для проведения анализа были выделены три набора данных: пожары, имеющие наблюдение только по данным VIIRS (пропущенные MODIS), пожары, имеющие наблюдение по данным MODIS (пропущенные VIIRS), и пожары, имеющие наблюдения по обоими источникам данных.

На Рис. 1 приведены графики общего количества пожаров в зависимости от площади и детектированных дополнительно при включении данных VIIRS в процедуры расчета пожаров.

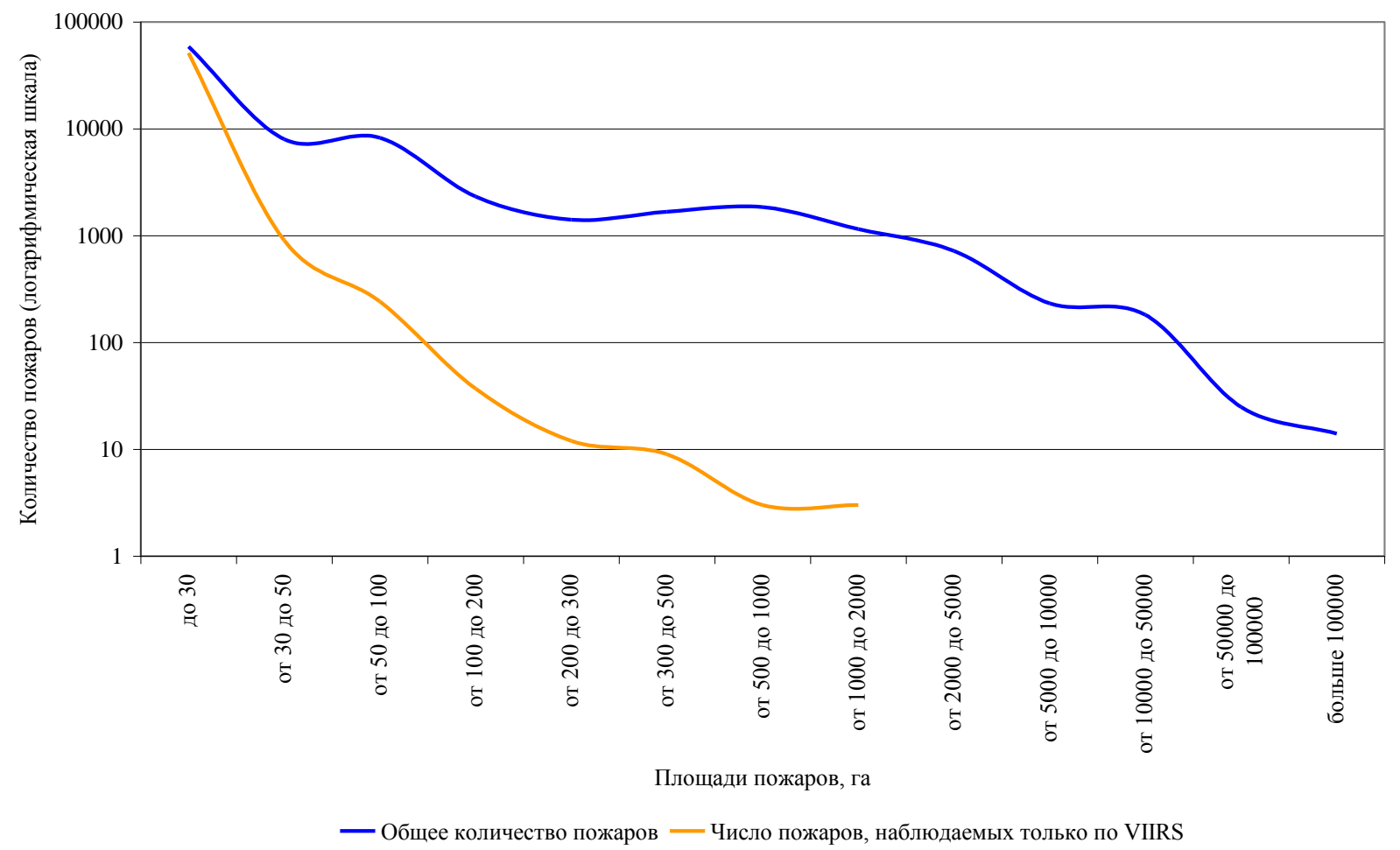

Рис. 1. Распределение количества наблюдаемых пожаров и детектированных дополнительно по данным VIIRS

Несмотря на большое абсолютное количество пожаров (61\% наблюдавшихся пожаров составляют пожары, сформированных только по данным VIIRS), их вклад в суммарные площади невелик. Площадь таких пожаров составляет только $2 \%$ от общей площади. При этом, площадь пожаров, детектированных только по данным MODIS, составляет 3\%. Это говорит о том, что, с одной стороны, данные MODIS хорошо подтверждены данными VIIRS, a с другой то, что использование «горячих точек» VIIRS позволяет детектировать большое количество пожаров с гораздо меньшей площадью чем детектируемые по MODIS. Средняя площадь детектированных только по данным VIIRS пожаров составила всего 8 га.

Для анализа влияния использования данных VIIRS на время регистрации пожаров была посчитана разница во времени от первого наблюдения по одному источнику до следующего наблюдения по другому источнику на основе набора пожаров, имеющие наблюдения и по данным VIIRS, и по данным MODIS. На основе этой информации получено распределение: до 34\% пожаров детектируются раньше по данным VIIRS, 35\% пожаров детектировано раньше по 
наблюдениям MODIS, оставшиеся пожары детектированы одновременно двумя источниками данных (с разницей во времени наблюдения до 30 минут). Таким образом, использование данных VIIRS позволяет детектировать раньше MODIS до 34\% пожаров. Распределение, показывающее преимущество во времени первого детектирования различных приборов приведено на рисунке 2.

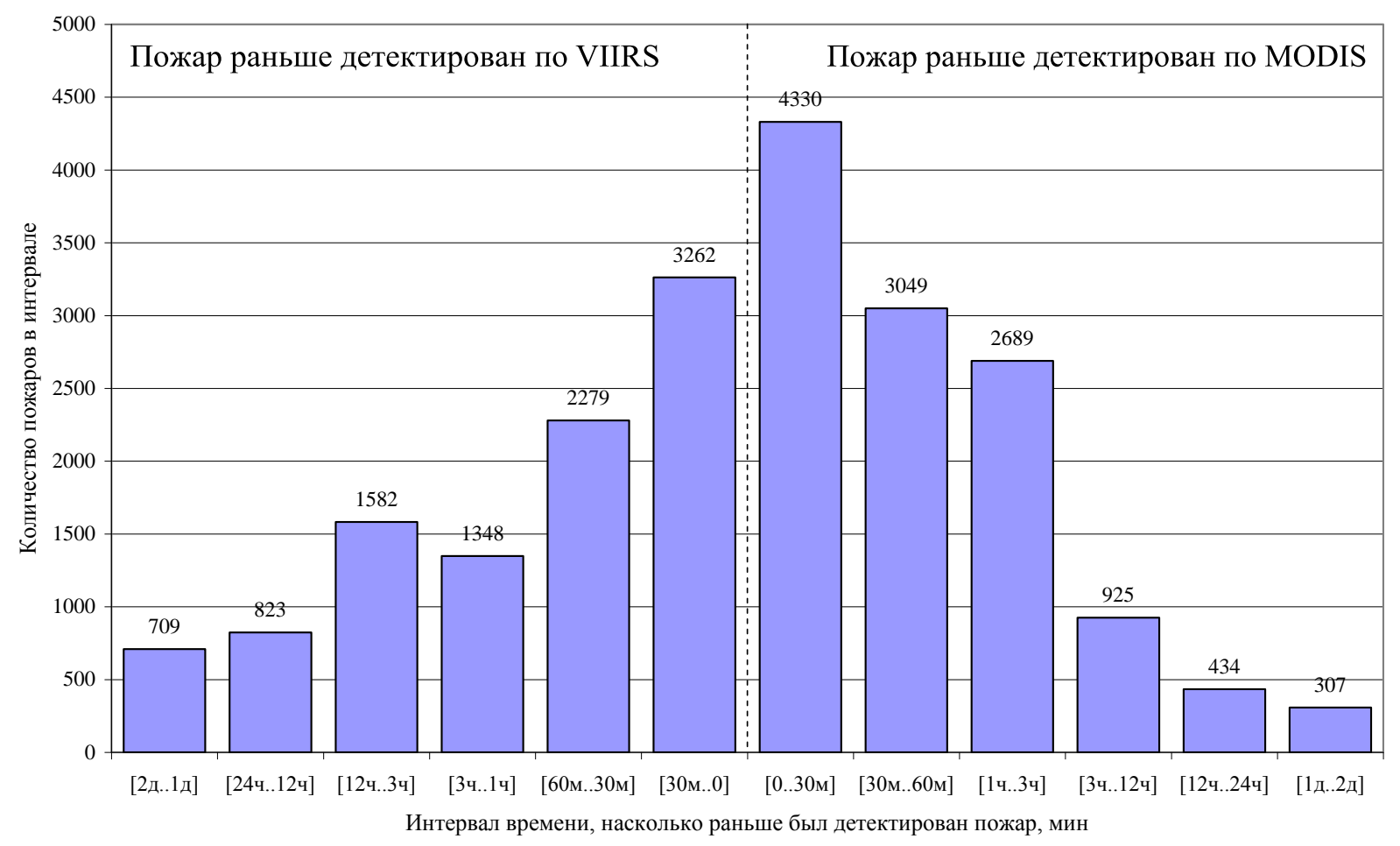

Рис. 2. Разницуа во времени наблюдения пожаров по данныл разных источников

Возвращаясь к количеству пожаров, детектированных только по данным VIIRS, необходимо ответить на вопрос - насколько достоверными являются наблюдаемые пожары. Для этого были использованы данные, вносимые в ИСДМ-Рослесхоз [1] региональными диспетчерскими службами субъектов РФ. Анализ комментариев и примечаний к пожарам, образованным только данными VIIRS показал, что субъекты подтвердили 90\% пожаров, что является высоким показателем достоверности.

\section{Выводы}

Информация о термических аномалиях, получаемая на основе данных прибора VIIRS, является достоверным и подтверждаемым наземными службами источником данных о пожарах. Использование данных VIIRS позволяет дополнительно регистрировать более $60 \%$ пожаров со средней площадью всего в 8 гектар. Включение информации со спутников NPP и NOAA-20 на вход блоков мониторинга пожаров информационных систем позволяет детектировать раньше до 34\% наблюдаемых совместно с MODIS пожаров, половина из которых - раньше от получаса до 3 часов, а 23\% наблюдалась раньше более чем за сутки.

Работа подготовлена в рамках государственного контракта Министерства науки и высшего образования Российской Федерации №05.577.21.0294 на тему: «Разработка технологий автоматизированной обработки спутниковых данных дистанционного зондирования Земли для создания и поддержки информационных сервисов мониторинга лесных ресурсов и охотничьих угодий России». Уникальный идентификатор проекта RFMEFI57718X0294. 


\section{References}

[1] Loupian E.A., Bartalev S.A., Ershov D.V., Kotelnikov R.V., Balashov I.V., Bourtsev M.A., Egorov V.A., Efremov V. Yu., Zharko V.O., Kovganenko K.A., Kolbudaev P.A., Krasheninnikova Yu. S., Proshin A.A., Mazurov A.A., Uvarov I.A., Stytsenko F.V., Sychugov I.G., Flitman E.V., Khvostikov S.A., Shulyak P.P. Satellite data processing management in Forest Fires Remote Monitoring Information System (ISDMRosleskhoz) of the Federal Agency for Forestry, Sovremennye Problemy Distantsionnogo Zondirovaniya Zemli iz Kosmosa, 2015, Vol.12, No 5, pp. 222-250. (In Russian).

[2] Loupian E.A., Bartalev S.A., Ershov D.V. Satellite Data Aplication Management in the ISDM-Rosleshoz System, Aerospace Methods and GIS-Technologies in Forestry, Forest Management and Ecology: Proceedings of the VI All-Russian Conference, Moscow, Russia, April 20-22, 2016. - M. CEPF RAS, 2016. P. 39-42. (In Russian).

[3] Bartalev S.A., Ershov D.V., Loupian E.A., Tolpin V.A., Vozmozhnosti ispol'zovaniya sputnikovogo servisa VEGA dlya resheniya razlichnykh zadach monitoringa nazemnykh ekosistem (Possibilities of Satellite Service VEGA Using for Different Tasks of Land Ecosystems Monitoring ), Sovremennye problemy distantsionnogo zondirovaniya Zemli iz kosmosa, 2012, Vol. 9, No. 1, pp. 49-56. (In Russian).

[4] URL: http://sozvezdie-vega.ru

[5] Loupian E.A., Bartalev S.A., Balashov I.V., Bartalev S.S., Bourtsev M.A., Egorov V.A., Efremov V. Yu., Zharko V.O., Kashnitskiy A.V., Kolbudaev P.A., Kramareva L.S., Mazurov A.A., Oksyukevich A.Y., Plotnikov D.E., Proshin A.A., Senko K.S., Uvarov I.A., Khvostikov S.A., Khovratovich T.S. Vega-Primorie: complex remote forest monitoring information system, Sovremennye Problemy Distantsionnogo Zondirovaniya Zemli iz Kosmosa, 2016, Vol. 13, No. 5, pp. 11-28. DOI: 10.21046/2070-7401-2016-13-5-11-28. (In Russian).

[6] Loupian E.A., Ershov D.V., Bartalev S.A., Isaev A.S. Information System for Remote Monitoring of Forest Fires and Their Consequences: The Results of the Last Decade and Further Prospects, Aerospace Method and GIS-Technologies in Forestry and Forest Management: Proceedings of the V All-Russian Conference, Dedicated to the Memory of V.Iv. Sukhikh and G.N. Korovin. Moscow, Russia, April 22-24, 2013.- M. CEPL RAS, 2013. P. 40-43. (In Russian).

[7] URL: https://ladsweb.modaps.eosdis.nasa.gov/missions-and-measurements/viirs/

[8] URL: https://modis.gsfc.nasa.gov/about/

[9] URL: https://lpdaac.usgs.gov/sites/default/files/public/product_documentation/mod14_atbd.pdf

[10] Stytsenko F.V., Loupian E.A., Bartalev S.A., Ershov D.V. Methods for estimating the area covered by forest fires based on satellite data of different spatial resolution, Sovremennye Problemy Distantsionnogo Zondirovaniya Zemli iz Kosmosa. Moscow, Russia, November 14-18 2016. pp. 396. (In Russian).

[11] URL: https://directreadout.sci.gsfc.nasa.gov/?id=dspcontent\&cid=68

[12] Bourtsev M.A., Milekhin O.E., Kramareva L.S., Antonov V.N., Balashov I.V., Kashnitskiy A.V., Loupian E.A., Matveev A.M., Proshin A.A., Uspensky S.A. Unified system of distributed work with the data of reception centers "SIC" Planeta ": current opportunities and development prospects, Sovremennye Problemy Distantsionnogo Zondirovaniya Zemli iz Kosmosa. Moscow, Russia, November 12-16 2018. pp 4. DOI: 10.21046/2070-16DZZconf-2018a. (In Russian).

[13] URL: https://firms.modaps.eosdis.nasa.gov/

[14] Flitman E.V., Balashov I.V., Bourtsev M.A., Galeev A.A., Egorov V.A., Kotelnikov R.V., Loupian E.A., Mazurov A.A., Matveev A.M., Proshin A.A. Organization of the MODIS Instrument Data Processing System for the Tasks of Monitoring Forest Fires and Their Aftereffects, Sovremennye Problemy Distantsionnogo Zondirovaniya Zemli iz Kosmosa, 2011, Vol. 8, No 1, pp. 127-138. (In Russian). 
[15] E.A. Loupian, A.A. Proshin, M.A. Burtsev, I.V. Balashov, S.A. Bartalev, V.Yu. Efremov, A.V. Kashnitskiy, A.A. Mazurov, A.M. Matveev, O.A. Sudneva, I.G. Sychugov, V.A. Tolpin, I.A. Uvarov. IKI center for collective use of satellite data archiving, processing and analysis systems aimed at solving the problems of environmental study and monitoring, Sovremennye problemy distantsionnogo zondirovaniya Zemli iz kosmosa, 2015, Vol.12, No 5, pp. 263-284. (In Russian). 